\section{Quantitative analysis of the} Dermott-Gold theory for Uranus's rings

DermotT and Gold ${ }^{1}$ have attempted to explain the locations of Uranus's rings in terms of resonances between ring particles and pairs of satellites, such that each particle librates about consecutive conjunctions of the satellites. The libration argument is given by

$$
K=\lambda_{1}-(p+1) \lambda_{2}+p \lambda_{3}
$$

where $\lambda_{\mathrm{i}}=n_{\mathrm{i}} t+$ constant, $i=1,2,3$, are the mean longitudes of the ring particle and the satellites, and $p$ is an integer. Despite the apparent success of the theory in predicting the main observed features of the rings, it is necessary to investigate the strength of the resonances in more detail before any conclusions can be drawn. This letter summarises the outcome of such an investigation which supplements the previous largely qualitative analysis ${ }^{1}$.

Aided by a study by Wilkens ${ }^{2}$ of possible three-body resonances involving one minor and two major planets, I derived an equation of motion analogous to that of a pendulum

$$
\frac{\mathrm{d}^{2} K}{\mathrm{~d} t^{2}}=c \cdot \sin K
$$

where $c$ is found to be roughly proportional to $m_{2} m_{3} \cdot\left(a_{2} / a_{3}\right)^{\mathrm{D}}$, that is the product of the masses of the two satellites times the ratio of their semi-major axes to the $p$-th power. In Table 1 , the first column gives the mass products adopted by Dermott and Gold for various pairs of satellites. The second column contains the range of values for $p=\left(n_{1}-n_{2}\right) /\left(n_{2}-n_{3}\right)$ required to span the observed width of the ring system-roughly 45,000 $52,000 \mathrm{~km}$. The corresponding range of $c$-values and of libration periods $\left(P=2 \pi \cdot c^{-\frac{1}{2}}\right.$ ) are given in the final two columns (the quoted periods are for zero-amplitude librations; the period increases slowly with the amplitude, being twice as large for $164^{\circ}$ as for $0^{\circ}$ ). Since $c>0$, librations can occur only about $K=180^{\circ}$.

On the basis of the magnitudes of the product $m_{2} m_{3}$ and the resulting ring pattern, Dermott and Gold concluded that the observed pattern is probably due primarily to the Ariel-Titania and Ariel-Oberon pairs. From the values of $c$, however, we now see that the correction factor $\left(a_{2} / a_{3}\right)^{\mathrm{p}}$ makes Miranda play the key role rather than Ariel, in spite of the small mass of the former. Furthermore, the single combination Miranda-Ariel dominates over all the others in terms of efficiency and predicts ring radii for $p=7,8$ and 9 which are very close to the radii obtained by Marsden ${ }^{3}$ for the $\varepsilon_{2}, \gamma$, and $\alpha$ rings, respectively. Therefore, when applied to this and other satellite pairs, the Dermott-Gold mechanism, as modified here, seems to have the capability of explaining the observed ring locations. It is not clear, though, how much statistical weight this argument should be given, one problem being that the model may predict too many rings - which, incidentally, provides hope for a future observational test. The relatively low $p$ values of the pairs involving Miranda may also add some new credibility to the theory. It is interesting to speculate that even lower values for $p$-and higher ones for $c$-would be attainable for a hypothetical pair of satellites involving Miranda and an as yet undiscovered satellite inside it. After all, the $80,000-\mathrm{km}$ wide gap between the rings and Miranda is not likely to be entirely empty.

On the other hand, the very long libration periods derived in Table 1, when compared with revolution periods of only about a third of a day for the ring particles, put very tight constraints on the semi-major axes of librating particles. A radial displacement of the order of $\Delta a_{1}=a_{1} \cdot \sqrt{ } c / n_{1} \leq 50 \mathrm{~m}$ is enough to disrupt the extremely weak particle-satellite-satellite coupling -and there are several competing forces that might set up a disturbance of that magnitude. Elliot et al. ${ }^{4}$ have estimated ring widths of from 12 to $85 \mathrm{~km}$ and particle sizes $<6 \mathrm{~km}$. The theory cannot account for these observations unless the particles are assumed to have slightly eccentric orbits. One important question not yet answered is whether the restoring force of the particle 'pendulum' is sufficiently strong to overcome the Poynting-Robertson effect for reasonable particle sizes.

\begin{tabular}{lccccc}
\hline \multicolumn{5}{c}{ Table 1 } & \multicolumn{5}{c}{ Ranges of $p, c$, and $P=2 \pi c^{-\frac{1}{2}}$} \\
\hline \multicolumn{1}{c}{ Satellites } & $m_{2} m_{3} \times 10^{11}$ & $p$ & $c(1,000 \mathrm{yr})^{-2}$ & $P(1,000 \mathrm{yr})$ \\
Miranda, Ariel & 19 & $7-9$ & $53-18$ & $0.87-1.5$ \\
Miranda, Umbriel & 5 & $4-6$ & $5.0-1.1$ & $2.8-5.9$ \\
Miranda, Titania & 32 & $3-5$ & $6.9-0.69$ & $2.4-7.5$ \\
Miranda, Oberon & 25 & $3-4$ & $1.8-0.32$ & $4.7-11$ \\
Ariel, Umbriel & 99 & $15-20$ & $4.6-0.73$ & $2.9-7.4$ \\
Ariel, Titania & 580 & $8-11$ & $2.1-0.15$ & $4.4-16$ \\
Ariel, Oberon & 447 & $7-10$ & $0.32-0.01$ & $11-62$ \\
Umbriel, Titania & 170 & $20-26$ & $(88-3.7) 10^{-4}$ & $67-320$ \\
Umbriel, Oberon & 131 & $15-19$ & $(63-2.1) 10^{-5}$ & $(2.5-14) 10^{2}$ \\
Titania, Oberon & 770 & $66-83$ & $(56-0.32) 10^{-8}$ & $(0.84-11) 10^{4}$
\end{tabular}

In conclusion, although sceptical of the Dermott-Gold theory, despite its ingenuity and elegance, I believe that a decisive test of the theory has to await further observational details of Uranus's rings. In the meantime, it would seem advisable to look for alternative ring theories. For example, Colombo (personal communication) points out that it may be of significance that the period associated with the $\varepsilon$ ring is in the approximate ratios $5: 4,6: 5,9: 8$, and $11: 10$ to those associated with the $\alpha, \beta, \gamma$, and $\delta$ rings. One should not discount the possibility, either, that the rings may be transient features not locked into any kind of resonance at all.

This work was supported by NASA grant NGR 09-015-213.

KaARe Aksnes

Center for Astrophysics,

Harvard and Smithsonian Observatories,

Cambridge, Massachusetts 02138

Received 5 August; accepted 31 August 1977

. Dermott, S. F. \& Gold, T. Nature 267, 590-593(1977).

Wilkens, A., \& Sitzungsber, D., Math-Natn. Abt. Bayer, D., Akad. Wiss, D. Zu Munchen, 1, 71-101 (1933).

Marsden, B. G. IAU Circ. No. 3061 (1977)

4. Elliot, J. L., Dunham, E. \& Mink, D Nature 267, 328-330(1977).

\section{Revenge of tiny Miranda}

DERMOTT and Gold ${ }^{1}$ have proposed a resonance model for the rings of Uranus. They assume the rings are, in fact, ares composed of small particles librating about stable resonances determined by pairs of satellites, either Ariel and Titania or Ariel and Oberon. Dermott and Gold dismiss as insignificant resonances involving tiny Miranda. We report here that, by a wide margin, the strongest resonances are all associated with Miranda. Furthermore, we show that the hypothesis that the rings are made up of librating particles, while original and ingenious, is incorrect.

Before considering the quantitative analysis of the resonance model, we make two minor points. First, when allowance is made for the orbital motion (assumed prograde) of the ring material between occultations, it is found that the two occulting regions of each ring were physically only $35^{\circ}\left(\alpha\right.$ ring) to $46^{\circ}(\varepsilon$ ring) apart Thus, only short ares are required to fit the observations. Second, while large systematic errors may remain in the calculated absolute ring radii, the spacings between the rings are well determined and must be accurately predicted by a resonance theory. In Table 1 , the spacings predicted by Dermott and Gold are compared with those deduced by Elliot et $a l^{2}$ and Marsden ${ }^{3}$. The discrepancies seem to be larger than observational crror and exhibit no systematic trend.

We have calculated the strengths of the resonances between a ring particle in circular orbit and either a single satcllite or a pair of 